\title{
EEG-Based Control for Human-Computer Interaction
}

\author{
Gloria L. Calhoun and Grant R. McMillan \\ Fitts Human Engineering Division \\ Crew Systems Directorate \\ Armstrong Laboratory \\ Wright-Patterson Air Force Base, OH 45433-7022
}

\begin{abstract}
An interface whereby brain responses can control machines has been developed by the Armstrong Laboratory. This EEG-based control uses the magnitude of the steady-state visual evoked response (SSVER) as a control signal. The SSVER is identified and monitored using non-invasive scalp electrodes and advanced signal processing technology. With biofeedback, users learn to increase or decrease the magnitude of the SSVER to an evoking stimulus. These responses are translated into commands that control the operation of a physical device or computer program. After further development, this innovative interface could revolutionize human interaction with complex systems.
\end{abstract}

\section{Introduction}

With recent advances in signal processing technology, it is now possible to reliably track specific brain signals only a few microvolts in amplitude and recorded with electrodes located on the scalp. Electroencephalographic (EEG)-based control is emerging as an exciting new channel of communication for humancomputer interaction. This innovative use of brain signals has the long-range potential to revolutionize human interaction with complex systems. EEG-based control is the only candidate alternative control that does not require physical manipulation (of the hands, fingers, voice, eyes, and/or head). Additionally, control based on brain responses may be less constrained by environmental conditions. A direct brain interface which facilitates headup and hands-free system operation may reduce operator workload and increase the overall bandwidth of control in a wide range of applications. For aviation and maintenance operations, EEG-based control allows the operator's hands to remain on critical components. For industry, space, rehabilitation, and chemical defense scenarios, EEG-based control provides an alternative interface when conventional controls are less accessible. Although near-term rehabilitation and entertainment applications are probable, EEG-based control for complex systems will require $5-10$ years development.

Research over several decades has established the foundation for brain actuated control and provides the current basis for examining potential applications. This paper reviews the methods used to date, summarizes research conducted by the Air Force Armstrong Laboratory as part of its evaluation of alternative control technologies, and discusses some of the issues and implications for the application of EEG-based control.

\section{Background}

EEG-based control involves harnessing specific rhythms and patterns in the brain's electrical activity that represent synchronized activity of large neuron groups. Two general approaches have been used in translating the EEG into system control inputs: 1) analyzing an operator's naturally occurring EEG patterns to identify a specific response or 2) training an operator to exert voluntary control over some EEG response feature (e.g., amplitude) [1]. Each of these approaches is described in more detail below.

\section{Spontaneous EEG Interpretation}

This approach involves the application of pattern recognition algorithms to detect characteristics in the $E E G$ which are associated with specific body movements, eye fixations or voice utterances. No user training is required. Rather, effort is devoted towards defining the relevant EEG signals and then training the algorithms with repetitions of the movement, fixations, or utterances such that the system can reliably recognize the defined components or patterns in spontaneous EEG. The objective of the research to date is to develop algorithms 
which can predict the control action under investigation. With long term development, this approach may evolve into a true "thought-based interface."

To date, this approach has primarily been pursued by Gert Pfurtscheller and his colleagues at the Graz University of Technology in Austria [2,3]. Neural networks are used to recognize EEG patterns in the 10-12 and $30-40 \mathrm{~Hz}$ bands which precede specific body movements. After training with 100-200 repetitions of the body movement, movement prediction is possible with only one second of EEG data. Thus, the speed of the neural network analysis should allow near real-time operation and control. Improving recognition accuracy, however, remains a technological challenge. Pfurtscheller's off-line system achieved $89 \%$ accuracy in predicting button pushes with the left or right hand. Accuracy dropped to $70 \%$ when toe and tongue movement predictions were added. Accuracy drops further if the algorithm is trained on imagined, rather than actual movements.

\section{Self-regulation of EEG Responses}

Operant conditioning of numerous aspects of animal and human EEG and neural evoked responses has been successfully demonstrated in research spanning three decades [4,5]. Animal studies have demonstrated specific conditioning of brain activity, independent of skeletal muscle responses or other artifacts [5]. Biofeedback is now commonly accepted as a technique for shaping brain electrical behavior and enabling voluntary modification of an EEG response.

Voluntary control of the $8-12 \mathrm{~Hz}$ "mu" rhythm is under investigation at the Wadsworth Center for Laboratories and Research in New York by Jonathan Wolpaw and his colleagues [6,7]. $\mathrm{Mu}$ is recorded over the primary sensorimotor areas of the brain and responds in known ways during movement preparation. Their system is designed to use the recorded rhythm to control the position of a cursor on a computer monitor. Users are tasked with moving the cursor to contact targets that appear randomly at the top, bottom, or corners of the monitor. The cursor provides feedback to the user, as its direction and step size are based on the amplitude of the mu rhythm. For a single-axis task (targets appear at monitor's top or bottom), users correctly select the target on $80-95 \%$ of the trials, taking $2-6$ seconds to move the cursor. On a dual-axis control task (targets appear in one of monitor's four corners), mu rhythm signals from both cortical hemispheres are used in a more complex algorithm. On this more difficult task, users typically are able to select $40-70 \%$ of the targets.

The EEG-based control under investigation at the Armstrong Laboratory is also based on the self-regulation of an EEG response [1]. The magnitude of the steadystate visual evoked response (SSVER) serves as the control signal. This approach has been used to perform several different types of tasks (see next section). In each of the task paradigms investigated, two types of feedback are provided. First, progression through the task steps, as a result of the EEG-based control, can be observed. Second, near real-time feedback on the amplitude of the SSVER is provided in a separate display element. To date, only single-axis control has been attempted. This involves translating the SSVER amplitude into a binary control signal.

\section{Armstrong Laboratory EEG-based Control Evaluations}

\section{System Description}

Studies at the Armstrong Laboratory have focused on the ability to voluntarily control EEG responses to sinusoidally modulated light. This feedback system simultaneously controls the frequency of the evoking stimulus and provides the user with feedback on the strength of the EEG response at that reference frequency. The current system (Figure 1) uses fluorescent lights partially modulated at $13.25 \mathrm{~Hz}$ to evoke a steady-state response in the visual cortex. The signal used for control is the magnitude of the bipolar SSVER recorded over occipital sites $\mathrm{O} 1$ and $\mathrm{O} 2$ (left and right visual cortical hemispheres, respectively, according to the 10-20 International System for surface electrode placement). The EEG signal from the scalp electrodes is amplified and then synchronously processed by a lock-in amplifier system. The lock-in amplifier provides a continuous measure of the magnitude of the $13.25 \mathrm{~Hz}$ SSVER component of the EEG signal that is simultaneously provided to the subject feedback display and the device control algorithm. Control logic based on threshold and duration requirements is employed to permit stable control of an external device despite variability in the user's SSVER control. One implementation produces a control output when the SSVER remains above or below experimenterspecified threshold for $75 \%$ of the samples in a one-half second interval. These settings require the user to produce sustained changes in the SSVER; however, brief SSVER fluctuations do not interrupt system control. The threshold, duration and percentage parameters are adjustable for individual subjects and for specific device applications. Various forms of this EEG-based controller have been used for different task paradigms. 


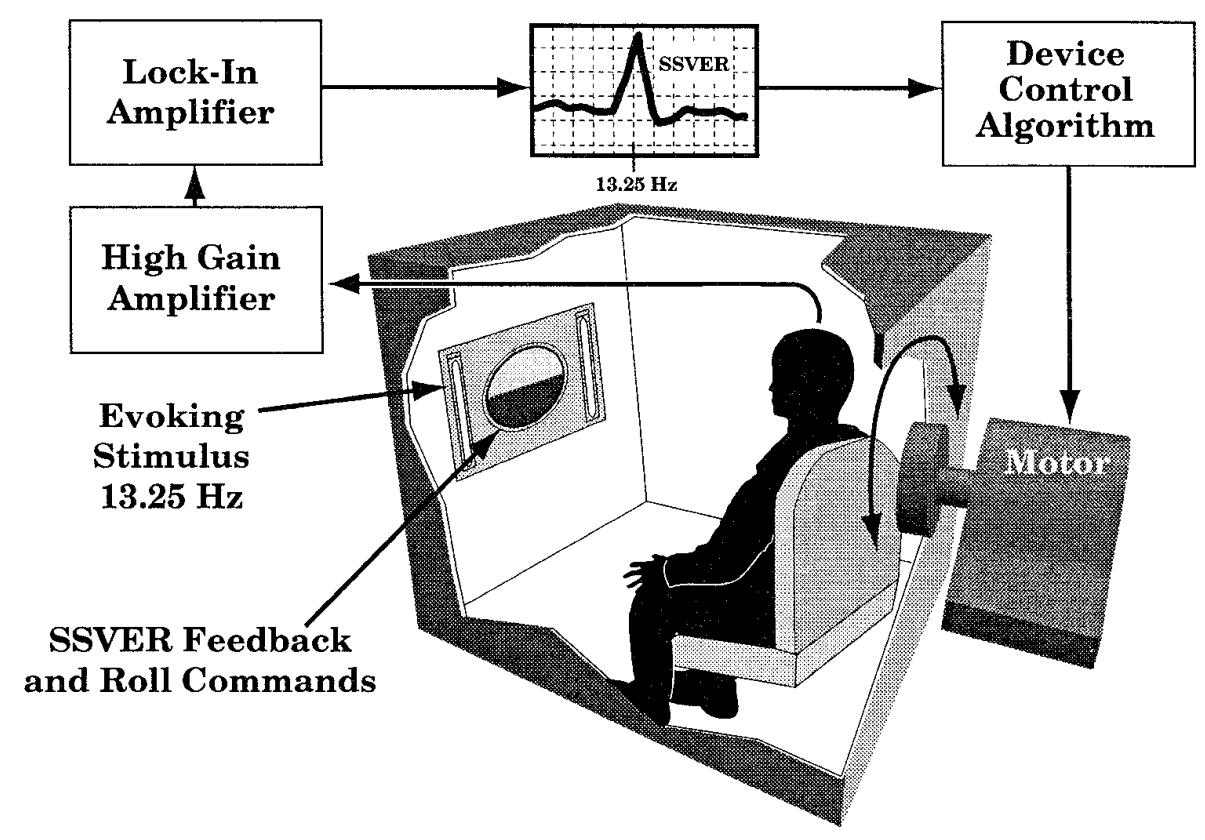

Figure 1. Schematic illustrating use of brain interface to control simulator roll position.

\section{Research/task Paradigms}

Earlier research in our laboratory focused on factors pertinent to implementing EEG-based control: stimulus frequency; effectiveness of feedback; effectiveness of different feedback modalities (visual, auditory, and kinesthetic); relationship between alpha production and effective lock-in amplifier loop closure; and factors affecting the speed of the visual-cortical response system [8-9]. Data were also collected to rule out the possibility of muscle biopotential contamination of the EEG response [8]. The lack of electromyographic (EMG) mediation is not surprising since this methodology is based on the control of very narrow band EEG activity. The results from these studies provide convincing evidence that people can achieve conscious control of their EEG response to stimuli of specific frequencies and that this ability can be used for device control. The Armstrong Laboratory has applied this EEG-based controller to a variety of task paradigms. Each of these is briefly described below, in the order they were implemented in our laboratory. Typical performance with each is also summarized.

Flight simulator roll position. With this task paradigm, the EEG-based interface is used to control the roll motion of a simple flight simulator [10; Figure 1]. A task display in the simulator provides a random series of commands requiring the user to roll right or left to specific target angles. As the user increases the SSVER magnitude above one threshold, the simulator rolls to the right. When the SSVER magnitude is decreased below a lower threshold value, the simulator rolls to the left.

A typical simulator control trial is presented in Figure 2. The user modulates the SSVER (as measured by the lock-in amplifier) in response to the roll command and the simulator moves in accordance with the output of the EEG interface. The operation of the control algorithm can be observed as simulator motion steps, separated by one-half second intervals. Typically, subjects are able to respond correctly to over $80 \%$ of the commands.

Muscle stimulator operation. SSVER selfregulation is used to operate a functional electrical stimulator (FES), a rehabilitation device designed to exercise paralyzed limbs [11]. Users are required to hold the SSVER magnitude above or below set thresholds for $85 \%$ of the samples in a one-second interval before a command is sent to the FES. Increasing SSVER magnitude above the "on" threshold causes the FES to activate at the muscle contraction level and begin ramping the current upward, gradually recruiting additional 


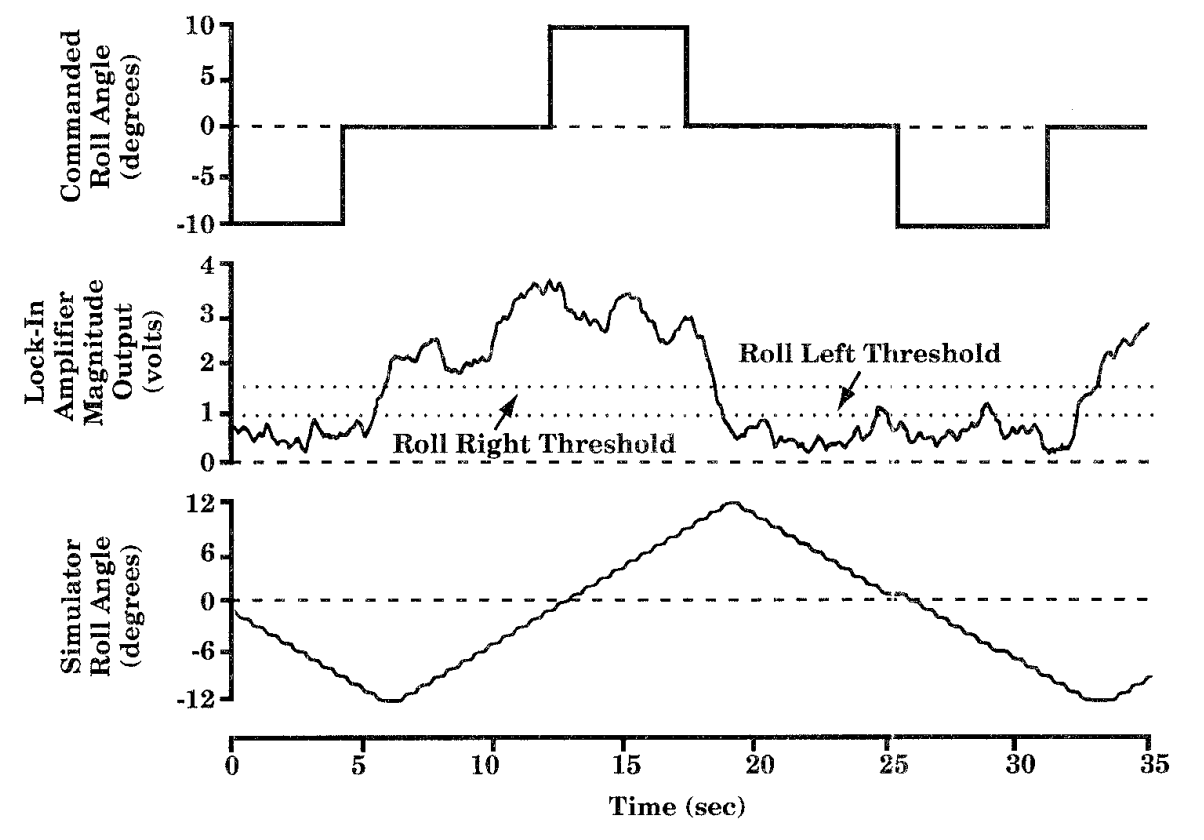

\section{Figure 2. Flight simulator control. Sample trial data. (Roll right designated positive, roll left designated negative.)}

muscle fibers to cause knee extension. SSVER magnitude below the lower "off" threshold results in the reversal of the FES system and subsequent ramp-down of the current.

In a pilot study, each of three trained able-bodied subjects participated in 3 to 5 one-hour sessions. Trial data were examined to ensure that there was a corresponding change in current level with each change in knee angle to confirm that the able-bodied subjects accomplished knee extension by controlling the brainFES interface. All three subjects were highly successful in repositioning the lower limb to match the commanded knee angle (95.8\% of the targets were acquired) and in returning the limb to its resting position.

Color matching task. This computer-based task involves matching the fill color of a square with that of its border [12]. The task display provides SSVER magnitude feedback and a randomly ordered series of red (enhance SSVER) or blue (suppress SSVER) color commands shown in the square's border. Users sustain SSVER enhancement or suppression above or below thresholds ( $100 \%$ of the samples for 2 seconds) to produce color steps in the corresponding direction of the commanded fill color. Each trial requires at least two successive correct color steps to match the command color.

The requirement to sustain the SSVER modulation for $100 \%$ of the samples in a two second interval is more demanding than the control parameters used in other task paradigms. These more stringent settings are used to facilitate collection of scalpwide broad band EEG for a parallel investigation of the topographic effects of SSVER self-regulation. To assist users in learning this more difficult task, initial sessions require sustained modulation for only $80 \%$ of the samples in a one second interval to produce a color step. As the subject acquires proficiency at this level, the threshold, duration and percentage requirements are gradually increased concomitant with performance improvements. After completing approximately 15 one-hour training sessions, subjects are typically able to match $75 \%$ of the commanded colors. The percentage of successful color matches would be even higher if the control parameters were less demanding. Although this control algorithm is not optimized for task performance, it si use demonstrates that subjects can sustain SSVER modifications for more than two seconds.

Switch selection. Users self-regulate the SSVER to select switch icons displayed on a computer monitor. Three "switches" (squares) are aligned next to three target fields on the display and the task involves selecting the switch next to the field containing a target (see Figure 3). To change which switch is selected, users enhance their SSVER above threshold to begin cycling through the switches. As soon as the SSVER meets threshold, the switch outline turns green. As the SSVER is sustained above threshold, the switch square outlined in green is 


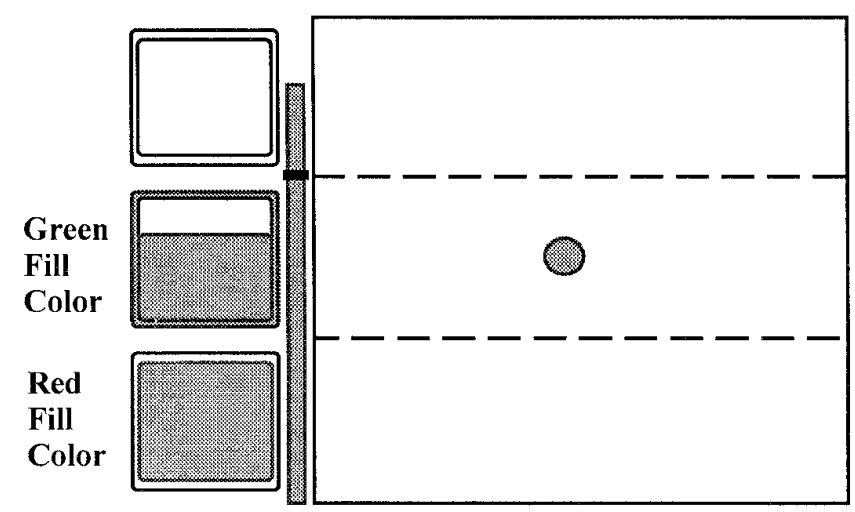

Figure 3. Schematic of switches, target field, and target used in switch selection task.

incrementally filled with green. Momentary drops below threshold halt, but do not reset, the fill process. Once the switch is entirely filled ( 1.5 second duration is met), the switch turns red and the next switch begins to fill. Maintenance of the SSVER above threshold continues the progression through the switches, moving vertically up the column. Once the top switch is filled, the progression "wraps around" to the bottom switch. To stop progression through the switches, the user must suppress the SSVER below threshold. The changes in the border and fill color of the switches provide feedback on the SSVER magnitude. In some implementations, a dynamic vertical bar is displayed between the switches and target fields to provide continuous SSVER magnitude information, relative to the threshold (shown in Figure 3 as hash mark between the top two switches).

Experimentation is underway using this task paradigm. Subjects are presented 12 blocks of 18 targets in 45 minute sessions. For each target, subjects are instructed to select the switch next to the field containing the target and pull the joystick trigger. Only preliminary data are available from three subjects; after eighteen sessions, the correct switch is typically selected for $70 \%$ of the targets.

\section{Some Implementation Issues}

Despite the success demonstrated in these laboratory task paradigms, several issues need to be addressed before EEG-based control can be used for human interaction with complex systems. To date, the control achieved can be described as rudimentary. Additional research is required to explore strategies for improving the dimensionality, accuracy, and bandwidth of control. Possibilities include the use of multiple EEG signals or patterns and the use of spatio-temporal patterns arising from the coordinated activity of multiple brain locations. Another promising approach would combine elements of the spontaneous
EEG recognition and self-regulation approaches to EEGbased control. Here one would begin with an EEG response that is naturally associated with the desired operator control output. Movement preparation EEG patterns are an example. Then one would employ biofeedback and closed-loop control techniques to allow the operator to improve the reliability and speed of the EEG response in order to perform some task.

If EEG-based control ultimately lacks the resolution and reliability afforded by many conventional controls, there are still potential applications where its head-up, hands-free nature offers real advantages. Ideally, EEGbased control will be integrated into future complex systems as a supplement to conventional control, rather than a substitute. In this manner, the combined potential of conventional and evolving alternative controls can be exploited.

Application in complex systems will require confirmation that EEG-based control is possible under real-world workload conditions. Experience to date suggests that learning EEG-based control does not require any special skills, unique individual characteristics or extensive training. However, it's utility in multitask environments and with ongoing distractions has not been examined. Its application will also require the differentiation between intended and unintended control signals changes. EEG is effectively "on" all the time; there is not a natural way to engage brain signals as an input device, similar to grasping/releasing a mouse. Some type of consent response may be required whereby the user either activates/deactivates EEG control or signals the system to act on the last recorded EEG response.

Successful application of EEG-based control will require system components that are portable, flexible, convenient, and relatively unobtrusive. Regardless of which EEG-control system is utilized, size, weight and cost are not serious constraints. Personal computer systems of the 80486 class are sufficient to support 
required computations, although digital signal processing boards are sometimes used. Continued development of "dry" EEG electrode recording systems is desirable to make application more convenient.

\section{Conclusions}

An adaptive interface which modifies system states as a function of the user's neural state would eliminate much peripheral neural and muscle activity from the humansystem control loop. By monitoring central neural signals, the interface adapts in some optimal fashion and executes user control intentions. This capability would be especially useful in task environments where conventional controls are difficult, if not impossible, to employ. EEG-based control hardware/software development shows great promise for adaptive humancomputer interaction. Rudimentary one- and twodimensional control and simple item selection have been demonstrated with current laboratory systems. However, improvements in flexibility, precision and reliability of EEG-based control are required for application in complex systems. Applications with current technologies are probably limited to assistive devices for the physically challenged and input devices for entertainment systems. Moreover, human factors research examining EEG-based control applications in complex systems remains the key challenge and opportunity.

\section{References}

[1] McMillan, G.R., Eggleston, R.G., \& Anderson, T.R. (in press). Nonconventional controls. In G. Salvendy (Ed.), Handbook of human factors and ergonomics (2nd ed.). New York: John Wiley \& Sons.
[2] Pfurtscheller, G., Flotzinger, D., Mohl, W., \& Peltoranta, M. (1992). Prediction of the side of hand movements from single-trial multi-channel EEG data using neural networks. Electroencephalography and Clinical Neurophysiology, $\underline{82}$, 313-315.

[3] Pfurtscheller, G., Flotzinger, D., \& Neuper, C. (1994). Differentiation between finger, toe and tongue movement in man based on $40 \mathrm{~Hz}$ EEG. Electroencephalography and Clinical Neurophysiology, 90, 456-460.

[4] Rockstroh, B., Birbaumer, N., Elbert, T., \& Lutzenberger, W. (1984). Operant control of EEG and event-related and slow potentials. Biofeedback \& Self-Regulation, 9, 139-160. [5] Elbert, T., Rockstroh, B., Lutzenberger, W., \& Birbaumer, N. (1984). Self-regulation of the brain and behavior. New York: Springer-Verlag.

[6] Wolpaw, J.R., \& McFarland, D.J. (1994). Multichannel EEG-based brain-computer communication. Electroencephalography and Clinical Neurophysiology, 90, 444-449.

[7] Wolpaw, J.R., McFarland, D.J., Neat, G.W., \& Forneris, C.A. (1991). An EEG-based brain-computer interface for cursor control. Electroencephalography and Clinical Neurophysiology, 78, 252-259.

[8] Junker, A.M., Schnurer, J.H., Ingle, D.F., \& Downey, C.W. (1988). Loop-closure of the visual cortical response (AAMRL-TR-88-()14). Wright-Patterson Air Force Base, OH: Armstrong Aerospace Medical Research Laboratory.

[9] Peio, K.J., and Junker, A.M. (1983). Visually evoked response from sum of sines stimulation. IEEE International Conference on Systems, Man, and Cybernetics, 1093-1098.

[10] McMillan, G.R., Calhoun, G.L., Middendorf, M.S., Schnurer, J.H., Ingle, D.F., \& Nasman, V.T. (1995). Direct brain interface utilizing self-regulation of the steady state visual evoked response. Proceedings of the RESNA 18th Annual Conference (RESNA), 693-695.

[11] Calhoun, G.L., McMillan, G.R., Morton, P.E., Middendorf, M.S., Schnurer, J.H., Ingle, D.F., Glaser, R.M., \& Figoni, S.F. (1995). Functional electrical stimulator control with a direct brain interface. Proceedings of the RESNA 18th Annual Conference (RESNA), 696-698.

[12] Nasman, V.T., Ingle, D.F., \& Calhoun, G. (1995). Topographic and task-related effects of SSVER self-regulation [Abstract]. Psychophysiology, 32, S55. 